\title{
Karyomegalic interstitial nephritis
}

\author{
N. Bennani Guebessi ${ }^{1} \cdot$ M. Karkouri ${ }^{1}$
}

Received: 5 April 2015/Accepted: 15 April 2015/Published online: 24 November 2015

(C) Japanese Society of Nephrology 2015

\begin{abstract}
Karyomegalic interstitial nephritis is a singular type of progressive chronic interstitial nephritis. The pathogenesis of this disease is unknown. The present study reported the case of a 22-year-old man who presented with a long history of recurrent upper respiratory tract infection episodes secondary to bronchiectasis and with progressive renal failure. Renal biopsy revealed chronic tubulointerstitial nephritis and a surprisingly marked karyomegaly specifically of the tubular epithelium.
\end{abstract}

Keywords Interstitial nephritis - Karyomegaly $\cdot$ Renal failure

\section{Introduction}

Karyomegalic interstitial nephritis (KIN) was first reported by Burry [1]. An ample description of patients with history of recurrent respiratory infections and progressive renal failure was given in 1979 by Mihatsch et al. [2]. This rare disease is characterized by chronic tubulointerstitial nephritis associated with marked karyomegaly in the tubular epithelium. The pathogenesis of KTN is unknown.
N. Bennani Guebessi nis.bennani@gmail.com

1 Department of pathology, UHC of IBN RUSCHD, Casablanca, Morocco

\section{Case report}

A 22-year-old male patient presented with a long history of recurrent upper respiratory tract infection episodes secondary to bronchiectasis and requiring amoxicillin therapy. He was diagnosed to have chronic renal failure. He was referred to the university hospital for further management. There was no family history of kidney disease and no history of drug ingestion. Serology of Epstein-Barr Virus, $\mathrm{BK}$ virus and Cytomegalovirus was negative.

Due to unknown kidney failure a biopsy was performed. Specimen was examined by light microscopy using hematoxylin/eosin (H\&E), Periodic acid Schiff (PAS), Periodic acid silver methenamine (PASM) stains and Masson's trichrome stain. The sample of renal cortex contained 13 glomeruli, three of whom showed global sclerosis. Some of the viable glomeruli demonstrated minimal enlargement in the mesangial matrix material. The glomerular capillary loops are thin and delicate. Endothelial and mesangial cells are normal in number. Tubular epithelial cells revealed changes. Many of the lining tubular cells revealed giant nuclei with hyperchromatic appearance (Figs. 1,2). No mitoses were seen. Furthermore, the interstitial zone showed focal fibrosis with tubular atrophy accompanied by lymphocytes and plasma cells. Blood vessels were without anomalies. In immunofluorescence, IgG, IgA, IgM, C3, C1q and fibrinogen were negative. We found low proliferation index in immunohistochemical stain with Ki67 (Fig. 3).

\section{Discussion}

In this report, we describe a case of KIN in a young man presenting with chronic renal failure and history of recurrent respiratory disease. KTN is a rare disease which is 


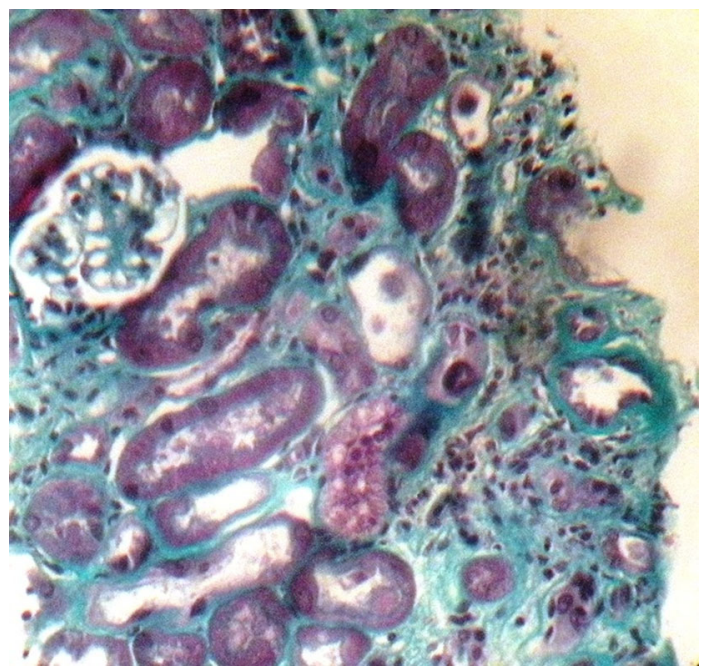

Fig. 1 Biopsy of the kidney shows nuclear enlargement and hyperchromasia tubular cell nuclei (Masson's trichrome $\times 400$ )

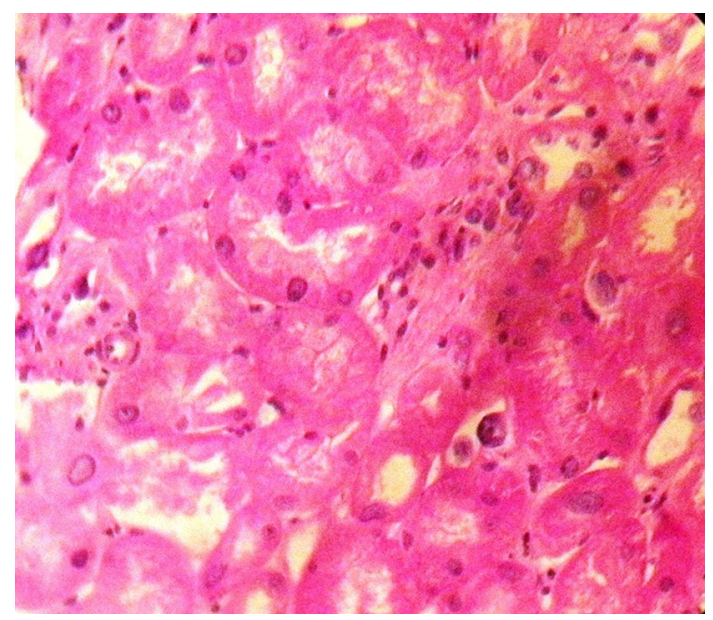

Fig. 2 Cortical tubules containing enlarged and pleomorphic nuclei $(\mathrm{H} \& \mathrm{E} \times 400)$

characterized by peculiar features: chronic tubulointerstitial nephritis and tubular nuclear changes like marked enlargement up to five- to sixfold in electron microscopy $(12-26 \mu \mathrm{m})$ [3], irregular outlines, course chromatin without viral inclusions. Extra-renal manifestations are rare, albeit karyomegalic cells have been detected in many tissues including brain astrocytes, smooth muscle cells in intestinal wall and arteries, acinar cells of pancreas, Schwann cells of peripheral nerves, Kupffer cells of liver and bile duct epithelium [3]. The prevalence of this disease remains less than $1 \%$ of all biopsies [4]. Pathogenesis of this disease is unknown. Mihatsch et al. [2] suggested that chemical toxins or viral infections are a cause of this disorder. In a report by Hassan et al. [5], high concentration of

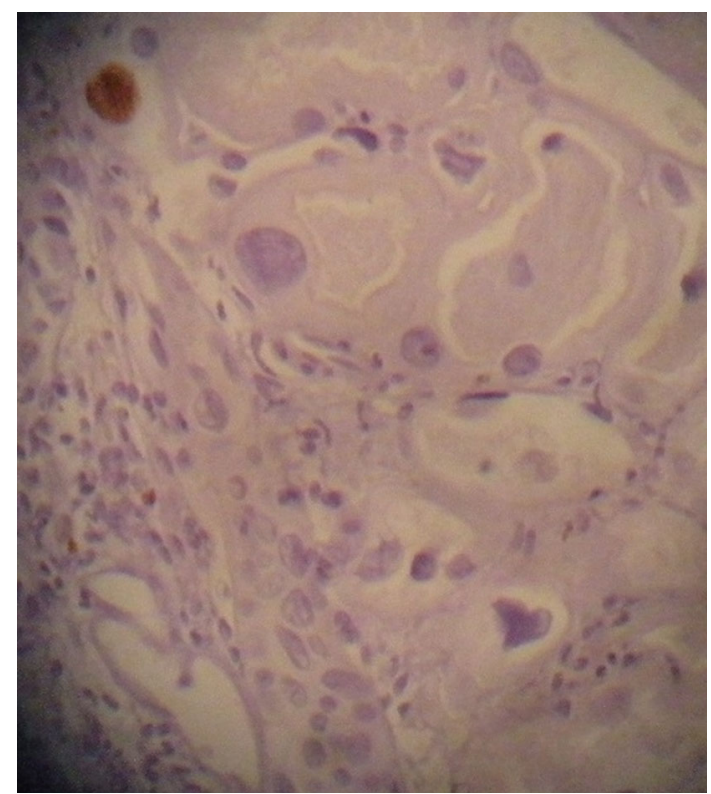

Fig. 3 Rare large nuclei were positive for Ki-67, but negative large nuclei were also observed $(\times 400)$

ochratoxin was found in the blood of affected siblings. McCulloch et al. [6], reported KIN in three adolescent patients treated with ifosfamide for Ewings sarcoma. Tomokazu Matsuura et al. reported a case of a 15-year-old boy presented with progressive nephrotoxicity 3 years after systemic chemotherapy with ifosfamide and cisplatin for the treatment of osteosarcoma. This case suggests the possibility that cisplatin may have a synergetic effect with ifosfamide for producing KIN [7].

Typically patients present with a history of recurrent upper respiratory infections and progressive renal failure in the third decade of life [2]. In our case, a long history of recurrent upper respiratory tract infection episodes needing amoxicillin was reported. In the literature review, there have been multiple published reports of amoxicillin associated with acute interstitial nephritis but the association of amoxicillin therapy with KNI was never observed.

Moreover, Spoendlin et al. studied Ki67 in tissue of four patients and concluded that there was inhibition of mitosis in karyomegalic cells. The hypothesis that the karyomegaly is secondary to a block in the G2 phase of the cell cycle has been proposed. In our case, Ki67 was estimated in the tubular epithelium with very low proliferation index. Major histocompatibility complex (MHC) typing revealed the A9/ B35 haptotype, which suggested a genetic defect in chromosome 6 that was linked to the MHC locus [8]. Godin et al. [9] reported two cases of karyomegalic interstitial nephritis in siblings with ochratoxin and HLA haplotype A9/B35. 


\section{Conclusion}

Karyomegalic interstitial nephritis is a peculiar disease often underdiagnosed. It is important to diagnose this pathology as it is a progressive disorder that causes an irreversible chronic lesion.

Conflict of interest The authors declare that they have no conflicts of interest.

\section{References}

1. Burry AF. Extreme dysplasia in renal epithelium of a young woman dying from hepatocarcinoma. J Pathol. 1974;113:147-50.

2. Mihatsch MJ, Gudat F, Zollinger HU, et al. Systemic karyomegaly associated with chronic interstitial nephritis. A new disease entity? Clin Nephrol. 1979;12(2):54-62.

3. Bhandari S, Kalowski S, Collett P, et al. Karyomegalic nephropathy: an uncommon cause of progressive renal failure. Nephrol Dial Transplant. 2002;17(11):1914-20.
4. Uz E, Bayram Y, Haltas H, Bavbek N, Kanbay M, Guz G, et al. Karyomegalic tubulointerstitial nephritis: a rare cause of chronic kidney disease. Nephrourol Mon. 2011;3:201-3.

5. Hassen W, Abid-Essafi S, Achour A, et al. karyomegaly of tubular kidney cells in human chronic interstitial nephropathy in Tunisia: respective role of ochratoxin $\mathrm{A}$ and possible genetic predisposition. Hum Exp Toxicol. 2004;23:339-46.

6. McCulloch T, Prayle A, Lunn A, Watson AR. Karyomegalic-like nephropathy, Ewing's sarcoma and ifosfamide therapy. Pediatr Nephrol. 2011;26:1163-6.

7. Matsuura $\mathrm{T}$, Wakino $\mathrm{S}$, Yoshifuji $\mathrm{A}$, et al. Improvement in karyomegalic interstitial nephritis three years after ifosfamide and cisplatin therapy by corticosteroid. CEN Case Rep. 2014;3:226-31.

8. Spoendlin M, Moch H, Brunner F, et al. Karyomegalic interstitial nephritis: further support for a distinct entity and evidence for a genetic defect. Am J Kidney Dis. 1995;25:242-52.

9. Godin M, Franck Le Roy A, Morin J-P, et al. Karyomegalic interstitial nephritis. Am J Kidney Dis. 1996;27(1):166. 